\title{
Deficiency of ATP13A2 Leads to Lysosomal Dysfunction, $\alpha$-Synuclein Accumulation, and Neurotoxicity
}

\author{
Marija Usenovic, ${ }^{1,2}$ Emilie Tresse, ${ }^{3}$ Joseph R. Mazzulli, ${ }^{1}$ J. Paul Taylor, ${ }^{3}$ and Dimitri Krainc ${ }^{1}$ \\ ${ }^{1}$ Department of Neurology, Massachusetts General Hospital, Harvard Medical School, Boston, MassGeneral Institute for Neurodegenerative Disease, \\ Charlestown, Massachusetts 02129, ${ }^{2}$ Mediterranean Institute for Life Sciences, Split 2100, Croatia, and ${ }^{3}$ Department of Developmental Neurobiology, St. \\ Jude Children's Research Hospital, Memphis, Tennessee 38105
}

The autophagy-lysosomal pathway plays an important role in the clearance of long-lived proteins and dysfunctional organelles. Lysosomal dysfunction has been implicated in several neurodegenerative disorders including Parkinson's disease and related synucleinopathies that are characterized by accumulations of $\alpha$-synuclein in Lewy bodies. Recent identification of mutations in genes linked to lysosomal function and neurodegeneration has offered a unique opportunity to directly examine the role of lysosomes in disease pathogenesis. Mutations in lysosomal membrane protein ATP13A2 (PARK9) cause familial Kufor-Rakeb syndrome characterized by earlyonset parkinsonism, pyramidal degeneration and dementia. While previous data suggested a role of ATP13A2 in $\alpha$-synuclein misfolding and toxicity, the mechanistic link has not been established. Here we report that loss of ATP13A2 in human fibroblasts from patients with Kufor-Rakeb syndrome or in mouse primary neurons leads to impaired lysosomal degradation capacity. This lysosomal dysfunction results in accumulation of $\alpha$-synuclein and toxicity in primary cortical neurons. Importantly, silencing of endogenous $\alpha$-synuclein attenuated the toxicity in ATP13A2-depleted neurons, suggesting that loss of ATP13A2 mediates neurotoxicity at least in part via the accumulation of $\alpha$-synuclein. Our findings implicate lysosomal dysfunction in the pathogenesis of Kufor-Rakeb syndrome and suggest that upregulation of lysosomal function and downregulation of $\alpha$-synuclein represent important therapeutic strategies for this disorder.

\section{Introduction}

Accumulation of misfolded proteins represents a pathologic hallmark of neurodegeneration and suggests that inadequate degradation of aggregation-prone proteins plays an important role in the pathogenesis of these disorders. In Parkinson's disease (PD) and other synucleinopathies, accumulation of $\alpha$-synuclein $(\alpha-$ syn) in Lewy bodies is a pathological hallmark of disease (Spillantini et al., 1997). Missense mutations (A30P, A53T, E46K) and multiplications of the $\alpha$-syn gene, in familial PD, enhance the propensity of $\alpha$-syn to aggregate (Conway et al., 2000; Singleton et al., 2003) and overexpression of $\alpha$-syn protein in various animal models of PD results in accumulation and neurotoxicity of $\alpha$-syn (Masliah et al., 2000; Auluck et al., 2002; Cao et al., 2005). These findings suggest that accumulation of $\alpha$-syn plays an important role in the pathogenesis of synucleinopathies and highlight $\alpha$-syn clearance as a key therapeutic target.

Increasing evidence implicates the autophagy-lysosomal pathway in mediating degradation of accumulated proteins in

Received Nov. 4, 2011; revised Jan. 24, 2012; accepted Feb. 13, 2012.

Author contributions: M.U. and D.K. designed research; M.U., E.T., and J.R.M. performed research; M.U., E.T., J.R.M., J.P.T., and D.K. analyzed data; M.U. and D.K. wrote the paper.

This work was supported by NIH Grant R01NS051303 (to D.K.). J.P.T. and E.T. were supported by NIH Grant R01AG031587. We thank Drs. Christine Klein for patient fibroblasts, Carolyn M. Sue for ATP13A2-GFP construct, Thomas J. Diefenbach for assistance with confocal microscopy, and Isabella Palazzolo, Hyunkyung Jeong, and other members of Krainc laboratory for helpful suggestions and discussions.

Correspondence should be addressed to Dr. Dimitri Krainc, MassGeneral Institute for Neurodegenerative Disease, 114 16th Street, Room 2008, Charlestown, MA 02129. E-mail: krainc@helix.mgh.harvard.edu.

DOI:10.1523/JNEUROSCI.5575-11.2012

Copyright $\odot 2012$ the authors $\quad 0270-6474 / 12 / 324240-07 \$ 15.00 / 0$ neurodegenerative diseases (Nedelsky et al., 2008; Nixon et al., 2008). While clearance of normal $\alpha$-syn is likely mediated by the ubiquitin-proteasome system (Webb et al., 2003), recent evidence suggests that autophagy-lysosomal pathway mediates clearance of accumulated and aggregated $\alpha$-syn (Cuervo et al., 2004; Mazzulli et al., 2011). Lysosomal dysfunction has been further implicated in the pathogenesis of synucleinopathies by identification of loss-of-function mutations in a gene for lysosomal P-type ATPase named ATP13A2 (PARK9) that causes KuforRakeb syndrome (KRS), a juvenile and early-onset parkinsonism with pyramidal degeneration and dementia (Ramirez et al., 2006; Park et al., 2011). Overexpression of wild-type ATP13A2 was shown to suppress $\alpha$-syn-mediated toxicity in yeast and Caenorhabditis elegans (Gitler et al., 2009; Yeger-Lotem et al., 2009), whereas ATP13A2 loss-of-function enhanced $\alpha$-syn misfolding in body wall muscle cells in a C. elegans model of PD (Hamamichi et al., 2008).

While previous studies suggested that ATP13A2 plays a role in $\alpha$-syn accumulation and toxicity, the mechanism has not been established. Here, we found that loss of ATP13A2 function resulted in impaired lysosomal function and consequently accumulation of $\alpha$-syn and neurotoxicity. Our findings further implicate lysosomal dysfunction in synucleinopathies and suggest that upregulation of lysosomal degradation capacity represents an important therapeutic target in PD and related disorders.

\section{Materials and Methods}

Plasmids. Lentiviral plasmids expressing short hairpin RNA (shRNA) targeting ATP13A2 and scrambled sequence control were in pLKO.1- 
puro vector backbone (Sigma-Aldrich). pLKO.1 plasmid with shRNA targeting $\alpha$-syn was purchased from Open Biosystems. Lentivirus was generated as previously described (Tiscornia et al., 2006) and virus titers were determined using HIV-1 p24 Antigen ELISA kit (Zeptometrix).

Fibroblasts and primary cortical neurons. Primary dermal fibroblasts from a male KRS patient carrying the $1550 \mathrm{C}>\mathrm{T}$ mutation in ATP13A2 (L6025) and sex-matched healthy control (WT1) were received from Christine Klein (University of Luebeck, Luebeck, Germany). Another sex-matched control (WT2) was obtained from American Type Culture Collection, CRL-2522. Mouse embryonic primary cortical neurons were prepared from E17 embryos of C56BL/6 mice as previously described (Jeong et al., 2009). Neurons were infected at a multiplicity of infection of 1 at day in vitro 7, and harvested $7 \mathrm{~d}$ postinfection (DPI) for immunostaining and Western. Leupeptin treatment (50 $\mu \mathrm{M}$, EMD Chemicals) was done $4 \mathrm{~d}$ before harvesting. Fibroblasts were transfected using Amaxa Basic Nucleofector kit (Lonza, VPI-1002).

Quantitative PCR. Total RNA was isolated from primary neurons at 7 DPI using Trizol reagent (Invitrogen) and treated with DNase (Qiagen). Reverse transcription was performed using SuperScript II First-Strand Synthesis SuperMix (Invitrogen) followed by quantitative PCR using SYBR GreenER SuperMix (Invitrogen) on the iCycler (Bio-Rad). Relative mRNA abundance was calculated by the $\Delta \Delta C \mathrm{t}$ method.

Western blots and immunofluorescence. Western blots were analyzed by Odyssey Infrared Imaging System (Li-Cor) and Odyssey software V2.1. Primary cortical neurons and fibroblast were lysed on ice in $1 \%$ Triton X-100 buffer and RIPA buffer, respectively, and resolved on 12 or $8 \%$ Tris-Glycine gels. Antibodies were anti- $\alpha$-syn 202 (Covance, 1:1000), anti LC3B (Cell Signaling Technology, 1:500), anti-EGF receptor (antiEGFR, Millipore, 1:1000), anti-human EGFR (anti-hEGFR, Cell Signaling Technology, 1:1000) anti-Tau (Dako, 1:1000). For immunofluorescence, cells were fixed in 4\% paraformaldehyde (PFA), permeabilized in blocking buffer ( $1 \times$ PBS, $4 \%$ goat serum, $0.1 \%$ BSA, $0.1 \%$ Triton X-100) for 1 h at RT and incubated with primary antibody at $4^{\circ} \mathrm{C}$. Antibodies were as follows: anti-LC3, 1:50 (Cell Signaling Technology); anti-LAMP1, 1:50 (Developmental Studies Hybridoma Bank and Santa Cruz Biotechnology). Fibroblasts were treated with LysoTracker Red DND-99 (Invitrogen, Molecular probes, 1:10,000 dilution) and neurons with LysoTracker Green DND-26 (Invitrogen, Molecular probes) following manufacturer's suggested protocol. Confocal microscopy was performed with Lieca TCS SL using $63 \times 1.4$ numerical aperture objective and live cell imaging with Zeiss LSM 510 META microscope with $25 \times$ objective. Quantitative analysis of fluorescence intensities were performed using Image (NIH) software.

Lysosomal degradation studies. Primary cortical neurons were treated with murine epidermal growth factor (EGF; Preprotech, $50 \mathrm{ng} / \mathrm{ml}$ ) on DPI 7 to stimulate endocytosis of epidermal growth factor receptor (EGFR), as monitored with anti-EGFR antibody (Liang et al., 2008). Fibroblasts were treated with $150 \mathrm{ng} / \mathrm{ml}$ of human EGF (Preprotech). The first-order rate constant $(k)$ of EGFR degradation was obtained by exponential decay fit. Long-lived protein degradation assay was performed by radioactive pulse-chase using $\left[{ }^{3} \mathrm{H}\right]$ leucine as previously described (Kaushik and Cuervo, 2009).

Monitoring autophagosome maturation. Generation of the mouse embryonic fibroblast cell line stably expressing mCherry-EGFP-LC3 was previously described (Tresse et al., 2010). RNAi knockdown were performed by transfection of ON-TARGET plus-Smartpool siRNA (Dharmacon, 050430 for ATP13A2 and 057592 for VCP; Thermo Fisher Scientific) with Lipofectamine RNAi Max (Invitrogen, 13778-075). The cells were imaged and red and yellow puncta quantified with a Marianas 2 confocal microscope using a $63 \times$ objective. All analyses were performed with Slidebook 5.0 software (Intelligent Imaging Innovations).

Neuronal toxicity. Toxicity was evaluated by LDH-Cytotoxicity assay Kit II (BioVision), following the manufacturer's protocol. Cortical neurons were seeded at 40,000/well in 96-well plate. Standard curve was generated using known concentrations of $\beta$-NAD-reduced disodium (Sigma, N6660). Neurofilament (NF) staining was performed with incell Western blot as previously described (Mazzulli et al., 2011). Cortical neurons were seeded in 96-well plate and fixed in 4\% PFA at DPI 5-12 followed by staining against neurofilament (anti-neurofilament, Developmental Studies Hybridoma Bank, University of Iowa, 1:1000).
Statistical analysis. Statistical analyses were calculated by two-tailed Student's $t$ test and Mann-Whitney rank sum test using SigmaPlot 11.0 software. Values are expressed as mean \pm SEM.

\section{Results}

\section{Loss of ATP13A2 results in accumulation and enlargement} of lysosomes

To explore the effect of loss-of-function of ATP13A2 on lysosomal pathway, we first examined PD patient fibroblasts harboring mutation $1550 \mathrm{C}>\mathrm{T}$ that presumably leads to retention of the mutant protein in the endoplasmic reticulum (ER; Ramirez et al., 2006; Park et al., 2011). Lysosomal profile was examined by lysosomal markers LAMP1 (lysosome-associated membrane protein-1), and LysoTracker. Immunofluorescence revealed a significant increase in the number and size of LysoTracker positive vesicles and LAMP1 immunostaining in patient fibroblasts compared with fibroblasts from healthy individuals, suggesting that lysosomes accumulate in the presence of ATP13A2 mutation (Fig. $1 A, B$ ).

To examine ATP13A2 function in primary cortical neurons, we developed a lentiviral transduction system to knockdown ATP13A2. Using this approach, $\sim 90 \%$ reduction of ATP13A2 expression was achieved on 7 DPI compared with scrambled shRNA control (Fig. $1 F$ ). Importantly, silencing of ATP13A2 in neurons resulted in increased number and size of LAMP1positive puncta when compared with scrambled shRNA (Fig. $1 C)$. Moreover, live cell imaging revealed an increase in the number of LysoTracker-positive puncta in ATP13A2 KD neurons compared with scrambled shRNA neurons (Fig. 1D). As additional control, we used leupeptin, a well established inhibitor of lysosomal proteases. Treatment of primary neurons with leupeptin resulted in a similar increase of LAMP1 and LysoTracker staining as seen in the presence of ATP13A2 knockdown (Fig. $1 C, D)$. Importantly, overexpression of wild-type ATP13A2 tagged with GFP (WT-ATP13A2-GFP), but not GFP alone, resulted in a significant decrease of LAMP1 staining in mutant fibroblasts (Fig. $1 E$ ). Together, these results demonstrate that the loss-of-function of ATP13A2 leads to upregulation of lysosomal size and number and that restoration of ATP13A2 can partially reverse these alterations.

\section{Loss of ATP13A2 leads to decreased lysosomal degradation in patient fibroblasts and primary neurons}

Having shown that loss of ATP13A2 function results in accumulation of lysosomes, we next tested whether lysosomal degradation capacity was affected. As a first step, we examined proteolysis of long-lived proteins in fibroblasts by radioactive leucine (L$\left[{ }^{3} \mathrm{H}\right]$ leucine). These experiments revealed significantly decreased proteolysis of long-lived proteins in patient fibroblasts compared with healthy controls (Fig. $2 \mathrm{~A}$ ). Treatment of cells with lysosomal inhibitors ammonium chloride $\left(\mathrm{NH}_{4} \mathrm{Cl}\right)$ and leupeptin did not exhibit additional decrease in the proteolysis indicating that lysosomal degradation pathway is primarily affected by loss of ATP13A2 (Fig. 2A).

To investigate whether loss of ATP13A2 function also compromises lysosomal degradation in neurons, we measured degradation of EGFR, an established readout of lysosomal degradation efficiency (Liang et al., 2008). Upon binding with EGF, EGFR is internalized and subsequently degraded in lysosomes. We found significantly decreased average EGFR degradation rate in ATP13A2-silenced neurons compared with shRNA scrambled controls (Fig. 2 B). Similarly, lower degradation rate of EGFR was observed in patient fibroblasts that carry mutant ATP13A2 (Fig. 


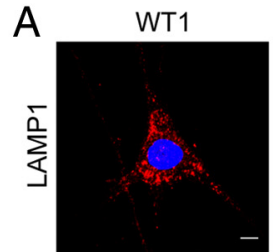

B

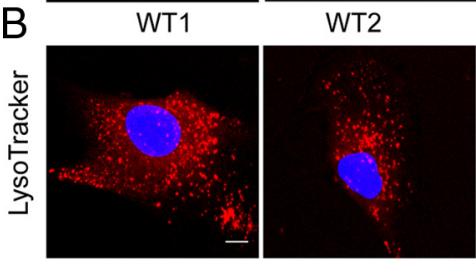

C

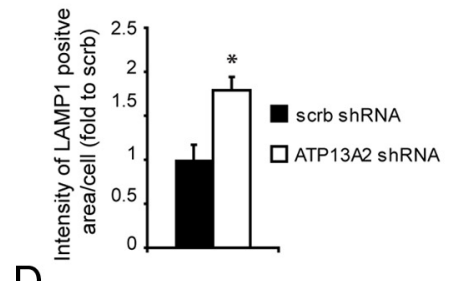

$\mathrm{D}$

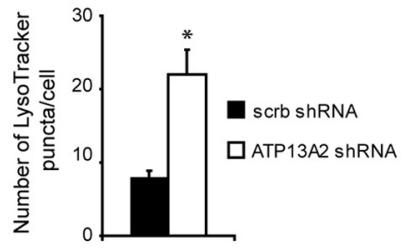

MUT

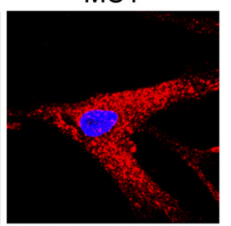

MUT
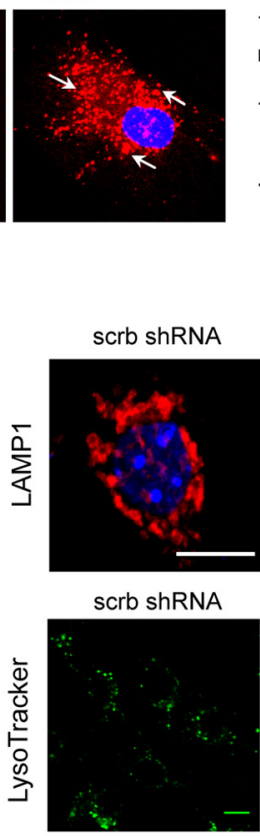
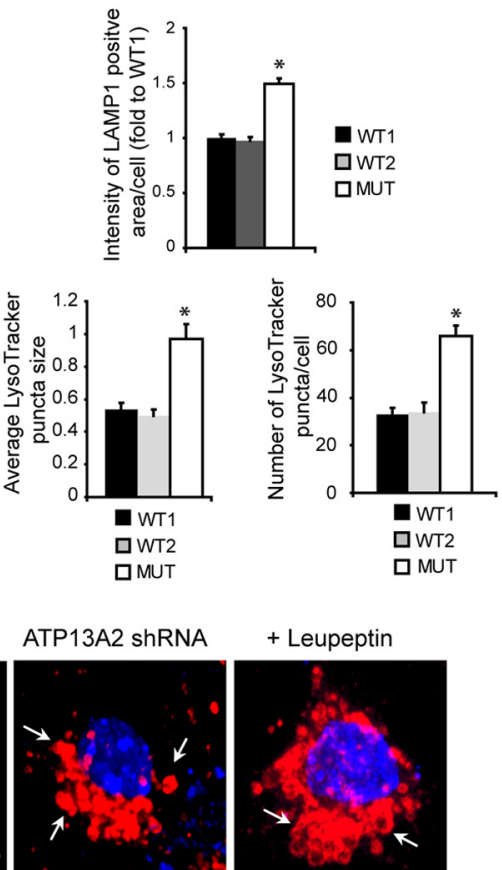

ATP13A2 shRNA

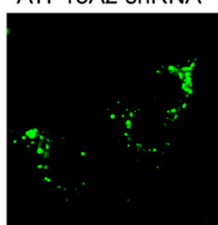

+ Leupeptin

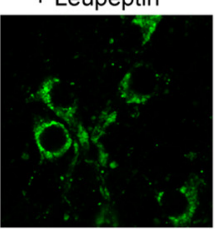

E

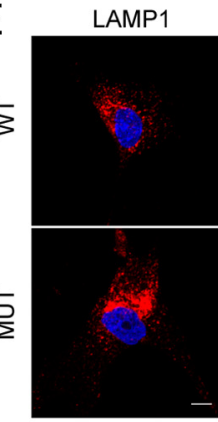

GFP
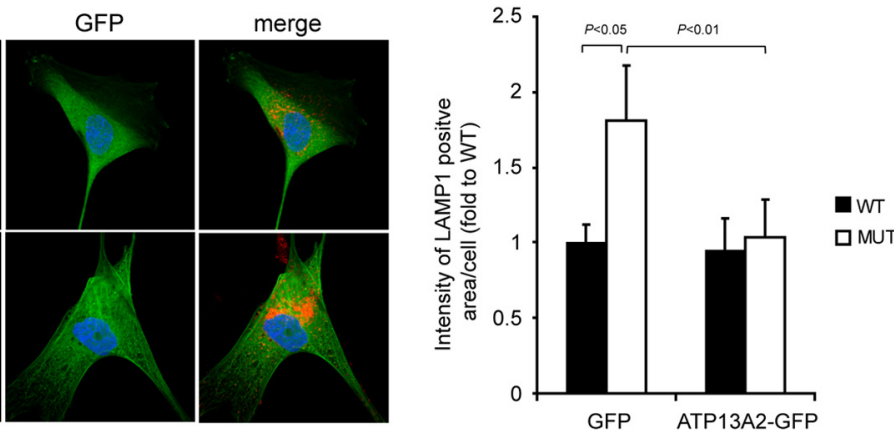

LAMP1

WT-ATP13A2-GFP

merge

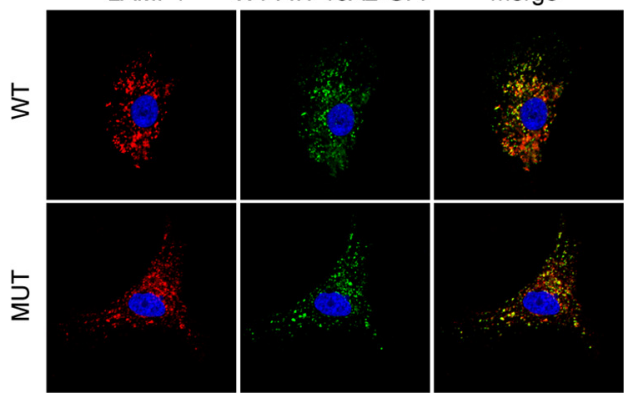

F

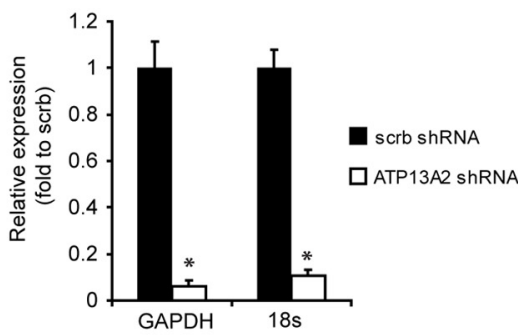

Figure 1. Accumulation of vesicles positive for lysosomal markers in ATP13A2 mutant fibroblasts and primary cortical neurons. $A$, Left, Representative images of LAMP1 immunostaining of two wild-type fibroblast lines (WT) and fibroblasts carrying ATP13A2 mutation (MUT); Right, quantification of intensity of cell area stained by LAMP1 $\left(n=80,{ }^{*} p<0.001\right)$. $\boldsymbol{B}$, Left, Imaging of LysoTracker labeled fibroblasts showed increased number and size of LysoTracker-positive vesicles, arrows indicate enlarged vesicles. Right, graphs: $n=80,{ }^{*} p<0.001$. C, Right, Representative images of LAMP1 immunostaining of primary cortical neurons. Fold change of LAMP1-positive area in ATP13A2 KD neurons compared with scrambled control cells; arrows indicate enlarged vesicles. Left, graph: $n=50,{ }^{*} p<0.001$. D, Right, Primary cortical neurons labeled with LysoTracker Green. Left, graph, quantification of the number of puncta $\left(n=25,{ }^{*} p<0.001\right)$. $E$, Left, Mutant or wild-type fibroblasts overexpressing GFP (top) or WT-ATP13A2-GFP (bottom) and immunostained with LAMP1. Right, Quantification of staining intensity is represented as fold change compared with WT line $(n=25) . \boldsymbol{F}$, Knockdown of ATP13A2 evaluated in cortical neurons by quantitative PCR. GAPDH and 185 were used as control genes $\left(n=3,{ }^{*} p<0.001\right)$. In all graphs, error bars indicate SEM. Scale bars, $10 \mu \mathrm{m}$. 
A
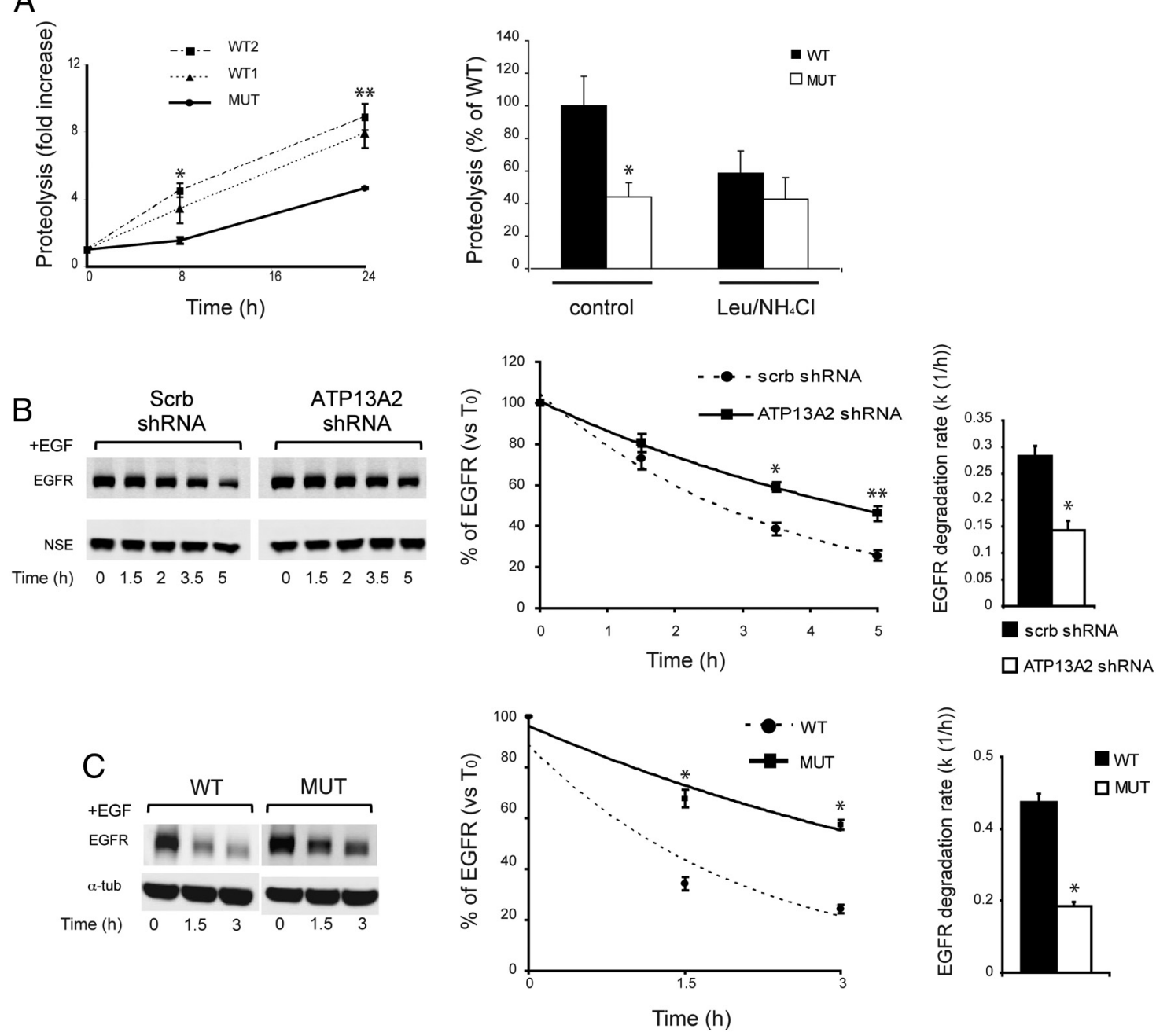

Figure 2. Decreased lysosomal degradation in ATP13A2-deficient neurons and patient fibroblasts. A, Left, Degradation of long-lived proteins in wild-type and mutant ATP13A2 fibroblasts at 8 and $24 \mathrm{~h}$ after incorporation of $\left[{ }^{3} \mathrm{H}\right]$ leucine $\left(n=4,{ }^{*} p<0.05,{ }^{* *} p<0.005\right)$. Values are expressed as fold increase from the initial time point. Right, The effect of leupeptin and ammonium chloride was examined at $8 \mathrm{~h}\left(n=4,{ }^{*} p<0.05\right)$. B, Left, EGFR degradation in primary cortical neurons was followed for $5 \mathrm{~h}$. Levels of EGFR were normalized to NSE levels and expressed as percentage levels of initial time point $\left(\mathrm{T}_{0} ; n=6,{ }^{*} p=0.019,{ }^{* *} p=0.008\right)$. Right, Average of degradation rates of EGFR is shown $\left([k(1 / \mathrm{h})] ; n=6,{ }^{*} p<0.001\right)$. C, EGFR degradation rate in patient fibroblasts compared with wild-type fibroblasts $\left(n=3,{ }^{*} p<0.001\right)$. In all graphs, error bars indicate SEM.

2C), further indicating that loss of ATP13A2 function leads to decreased lysosomal degradation capacity.

\section{Clearance of LC3-positive vesicles is impaired in cells depleted for ATP13A2}

Since our data so far suggest that lysosomal degradation capacity is decreased in the presence of mutant ATP13A2, we next examined the status of macroautophagy. Productive macroautophagy includes the lipidation of Atg8/LC3 and the formation of autophagosomes that fuse with lysosomes to release the breakdown products (Martinez-Vicente and Cuervo, 2007). As a first step, we examined LC3-II, a membrane-associated marker of autophagosomes (Kabeya et al., 2000) that is degraded by the lysosomes. Western blot analysis showed a significant increase in LC3-II to LC3-I ratio in ATP13A2 KD neurons compared with scrambled controls (Fig. 3A). Consistent with this, immunostaining revealed an increase in LC3positive puncta in KD neurons compared with control cells (Fig. $3 B$ ). Using leupeptin as additional control, a similar increase in LC3 staining was observed in leupeptin-treated neurons (Fig. 3B). These results suggest that silencing of ATP13A2 leads to accumulation of autophagosomes in neurons.
To examine the turnover of autophagosomes, we used mouse embryonic fibroblasts (MEFs) that stably express tandem-tagged fluorescence reporter mCherry-EGFP-LC3b. This reporter is useful for distinguishing immature autophagosomes (emitting yellow signal) from mature autophagolysosomes (emitting red signal; Tresse et al., 2010). This is because both mCherry and EGFP emit fluorescence in the neutral environment of the immature autophagosome, but EGFP fluorescence is quenched by the acidification that occurs upon fusion with lysosomes. MEF cells that were transfected with ATP13A2 siRNA exhibited a significantly increased number of immature autophagosomes (yellow puncta) and a decrease in the number of mature autophagolysosomes (red puncta) compared with cells transfected with nontargeting siRNA (Fig. 3C). As a positive control, we used siRNA-mediated $\mathrm{KD}$ of VCP/p97 in MEF cells that is known to cause accumulation of immature autophagosomes due to a failure in autophagosomelysosome fusion (Tresse et al., 2010).

These findings suggest that mutant ATP13A2 leads to impaired lysosomal clearance of autophagic vesicles, further confirming decreased lysosomal degradation capacity of cells that lack functional ATP13A2. 
A

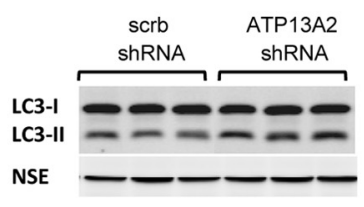

B

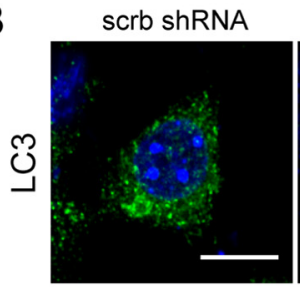

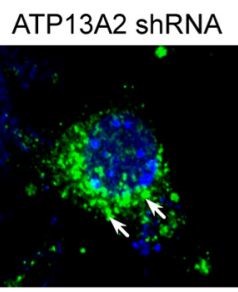

+ Leupeptin

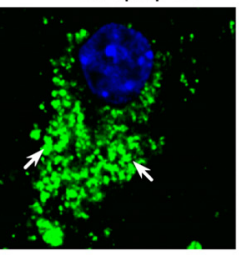

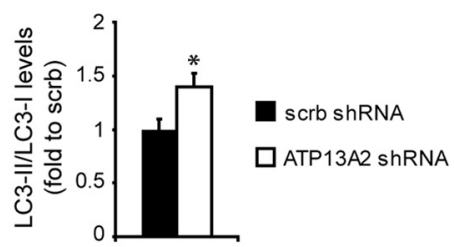
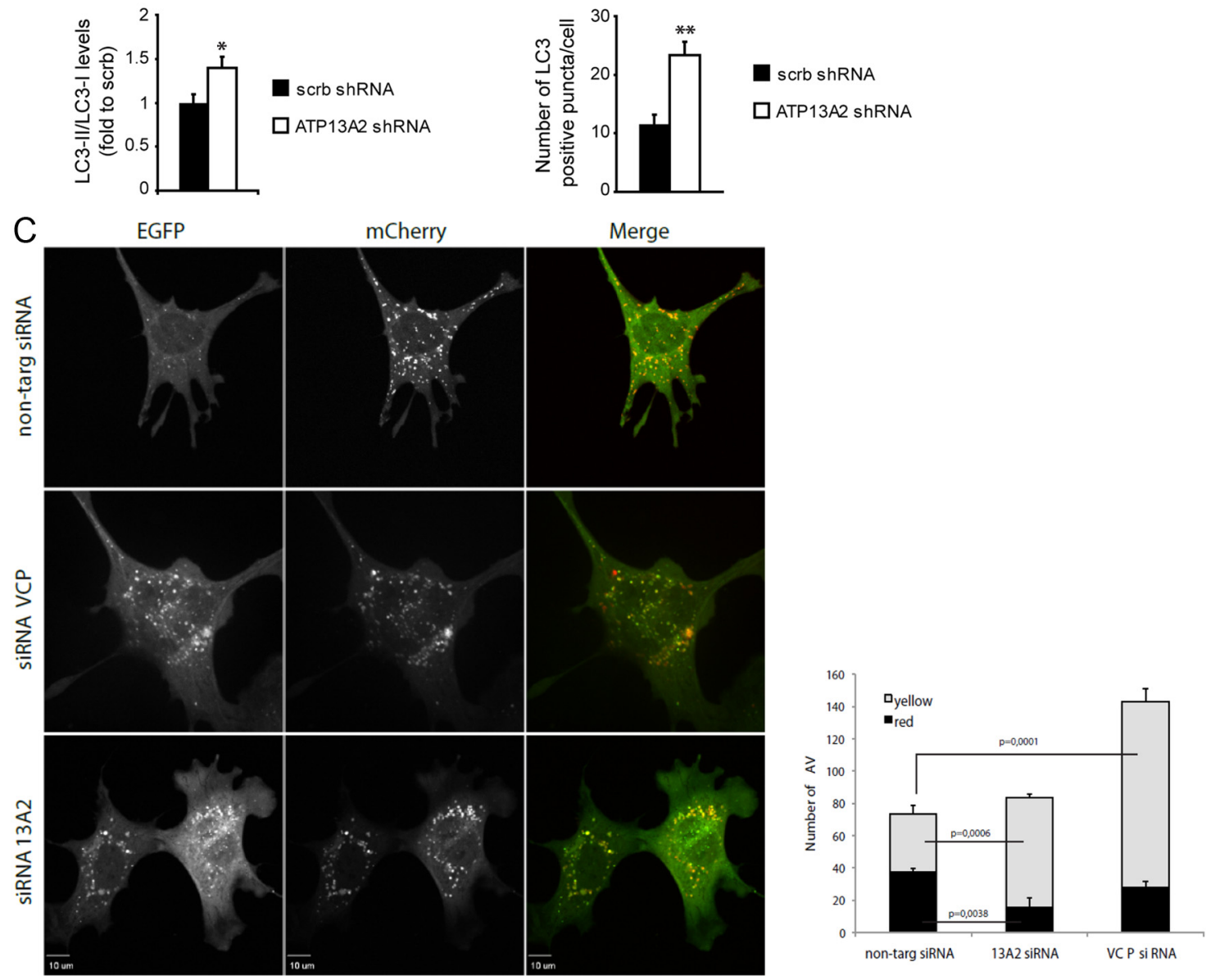

Figure 3. Depletion of ATP13A2 leads to increased LC3-positive vesicles. $A, \mathrm{LC} 3-\mathrm{II} / \mathrm{LC} 3-\mathrm{I}$ ratio is represented as fold change over scrambled (scrb) shRNA $\left(n=8,{ }^{*} p<0.05\right)$. NSE was used as loading control. $\boldsymbol{B}, \mathrm{LC} 3$-positive puncta in ATP13A2 shRNA neurons compared with scrb shRNA $\left(n=50,{ }^{* *} p<0.001\right)$. Leupeptin was used as positive control. Arrows indicate enlarged $L C 3$ vesicles. Scale bar, $10 \mu \mathrm{m}$. C, MEF cells stably expressing mCherry-EGFP-LC3b fluorescence reporter were transfected with siRNA for ATP13A2, nontargeting control and VCP/p97. Graph presents quantifications of autophagosomes (yellow puncta) and autophagolysosomes (red puncta). In all graphs, error bars indicate SEM from three independent replicates.

\section{Loss of ATP13A2 in neurons causes accumulation of $\alpha$-syn and toxicity}

Since previous results in C. elegans suggested that ATP13A2 is a modifier of $\alpha$-syn toxicity (Gitler et al., 2009), we examined whether deficient lysosomal function in ATP13A2 KD neurons leads to accumulation of $\alpha$-syn. Indeed, we found significantly increased levels of endogenous $\alpha$-syn in ATP13A2 KD compared with neurons expressing scrambled shRNA control (Fig. $4 A$ ). Interestingly, we did not observe an accumulation of another aggregation-prone protein tau (Fig. 4B), suggesting that decreased lysosomal function in ATP13A2 KD neurons preferentially affects $\alpha$-syn.

In light of the previous report demonstrating ATP13A2mediated rescue of $\alpha$-syn toxicity (Gitler et al., 2009), and our observation that loss of ATP13A2 leads to decreased lysosomal function and accumulation of $\alpha$-syn, we next examined whether silencing of ATP13A2 in primary cortical neurons resulted in toxicity. Neuronal toxicity was evaluated by nuclear condensation, NF staining and release of lactate dehydrogenase (LDH) into culture medium. These experiments revealed that knockdown of ATP13A2 in neurons resulted in decreased NF staining (Fig. 4C), increased release of LDH (Fig. $4 D$ ), and increased nuclear condensation (Fig. 4E, graph), suggesting that loss-of-function of ATP13A2 leads to neuronal toxicity. To examine whether the observed toxicity was dependent on the presence of accumulated $\alpha$-syn, we developed a lentiviral system to efficiently knockdown endogenous $\alpha$-syn in neurons by $>80 \%$ (Fig. $4 F)$. Using NF staining as readout, we found that in the presence of silenced endogenous $\alpha$-syn, toxicity was attenuated in ATP13A2depleted primary neurons, further suggesting that the neurotoxicity due to loss of ATP13A2 is at least in part mediated by the lysosomal dysfunction and consequently accumulation of $\alpha$-syn (Fig. $4 F$ ).

\section{Discussion}

Lysosomal dysfunction has been reported in several neurodegenerative disorders including PD, Alzheimer's disease, and amyo- 
A

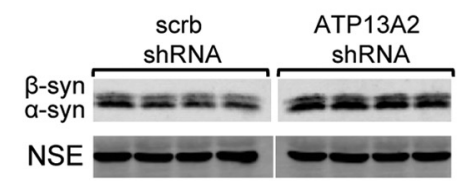

C

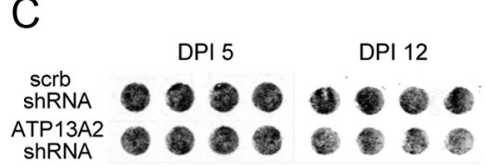

E

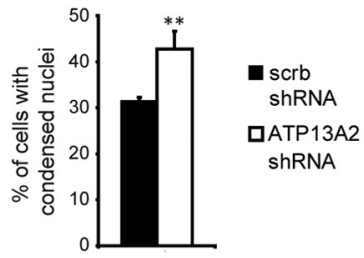

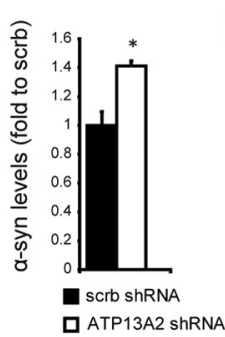

B

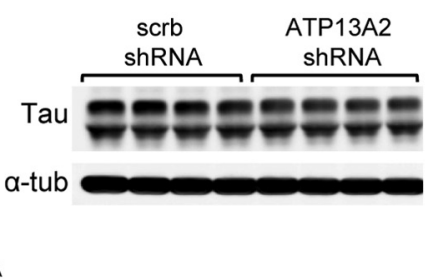

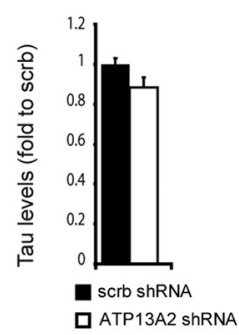

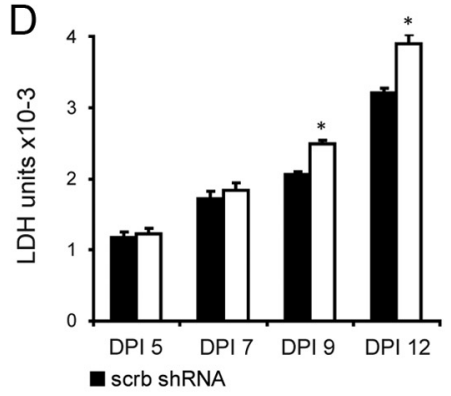

口ATP13A2 ShRNA

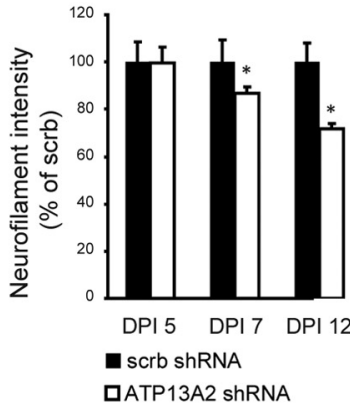

$\mathrm{F}$

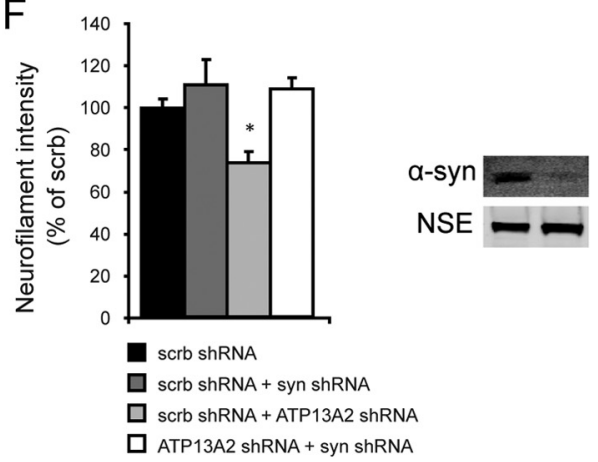

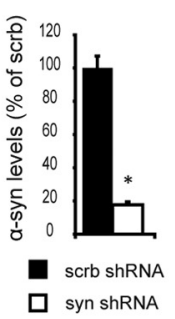

Figure 4. Depletion of ATP13A2 leads to accumulation of $\alpha$-syn and toxicity in primary neurons. $\boldsymbol{A}$, Levels of $\alpha$-syn protein normalized to NSE and presented as fold change over scrambled (scrb) shRNA levels ( $\left.n=6,{ }^{*} p=0.002\right)$. B , Western blot analysis of Tau protein levels in scrb shRNA and ATP13A2 shRNA; $\alpha$-tubulin was used as loading control ( $\left.n=10, p=0.578\right)$. $\boldsymbol{C}$, In-cell Western blot for neurofilament staining of neurons infected with lentivirus carrying ATP13A2 shRNA and scrb shRNA at DPI 5 and DPI 12. Graph shows quantification of neurofilament intensity expressed as a percentage of scrb shRNA $\left(n=4,{ }^{*} p<0.001\right)$. D, LDH activity in neuronal culture medium at indicated time points $\left(n=4,{ }^{*} p<0.001\right)$. $\boldsymbol{E}$, Percentage of neurons with condensed nuclei $[n$ (cells) $=180$, four different fields, ${ }^{* *} p<0.05$ ]. $\boldsymbol{F}$, Left, Quantification of neurofilament intensity of neurons with knockdown of $\alpha$-syn and knockdown of ATP13A2 ( $n=4$, ${ }^{*} p<0.001$ ). Right, Western blot showing silencing of endogenous $\alpha$-syn $\left(n=3,{ }^{*} p<0.001\right)$. In all graphs, error bars indicate SEM.

trophic lateral sclerosis (Nixon et al., 2008). Moreover, there is increasing evidence that patients with lysosomal storage diseases also exhibit neurodegeneration, further suggesting the importance of normal lysosomal function for neuronal survival (Schultz et al., 2011).

To more directly examine the link between lysosomes and neurodegeneration, it is particularly informative to study neurodegenerative diseases caused by mutations in genes implicated in lysosomal function. One such disease is Kufor-Rakeb syndrome that is characterized by early-onset parkinsonism with pyramidal degeneration and dementia (Williams et al., 2005). This syndrome is caused by mutations in lysosomal protein ATP13A2 that lead to retention of the mutant protein in the ER (Ramirez et al., 2006; Park et al., 2011). However, the consequences of this ER retention of mutant ATP13A2 have not been previously examined and postmortem brain tissues from these patients have not been available. We found that depletion of ATP13A2 in primary cortical neurons resulted in accumulation of lysosomes. Similar results were obtained in patient fibroblasts that contain endogenous mutant ATP13A2, suggesting that loss of ATP13A2 function accurately models disease pathogenesis. In addition, overexpression of normal ATP13A2 partially restored the normal lysosomal profile, further underscoring the importance of ATP13A2 function for lysosomal function. Although the precise reasons for lysosomal accumulation in our system remain unknown, we hypothesized that cellular compensation for defective lysosomal degradation could play a role. In support of this conclusion, we found lower rate of proteolysis of long-lived proteins and impaired degradation of EGFR. In addition, we found accumulation of autophagosomes in the presence of silenced ATP13A2, further suggesting deficient lysosomal degradation capacity. Importantly, diminished lysosomal degradation in ATP13A2-depleted neurons resulted in preferential accumulation of endogenous $\alpha$-syn, highlighting the importance of intact lysosomal function for turnover of $\alpha$-syn. Recent genetic and clinical studies point to a clear dosage relationship between $\alpha$-syn and disease. For example, duplications and triplications in SNCA gene result in increased expression of normal $\alpha$-syn and familial Parkinson's disease (Devine et al., 2011). Moreover, variations in SNCA locus that result in increased levels of $\alpha$-syn are the major genetic risk factor for sporadic disease (Devine et al., 2011). Although these studies strongly suggest that $\alpha$-syn accumulation plays a key role in the pathogenesis of synucleinopathies, the molecular mechanisms of $\alpha$-syn accumulation and clearance 
have not been well understood. Recent identification of mutations in lysosomal genes that are also linked to neurodegeneration has offered an opportunity to examine these pathways in more detail. To this end, we recently demonstrated that mutations in lysosomal enzyme glucocerebrosidase result in decreased lysosomal proteolytic function and accumulation of $\alpha$-syn (Mazzulli et al., 2011). While either deficiency of lysosomal glucocerebrosidase or ATP13A2 results in impaired lysosomal function, the mechanisms responsible for $\alpha$-syn accumulation are likely distinct. In the case of mutated glucocerebrosidase, there is accumulation of its lipid substrates that in turn directly alter $\alpha$-syn aggregation pathway (Mazzulli et al., 2011). The role of wild-type ATP13A2 is not known, but it is predicted to function as lysosomal P5-type ATPase that regulates cation homeostasis (Tan et al., 2011). Therefore, the mechanism responsible for the observed impairment of lysosomal degradation in our models of KuforRakeb syndrome remains unknown. However, our finding that the toxicity of ATP13A2-depleted neurons at least in part depends on the presence of endogenous $\alpha$-syn, suggests that lysosomal dysfunction and $\alpha$-syn accumulation play a key role in ATP13A2-mediated toxicity. The unique primary amino acid sequence of $\alpha$-syn, containing a stretch of mostly hydrophobic residues between positions 71-82, renders it more likely to form amyloid fibrils and aggregates compared with most other cellular proteins (Chiti and Dobson, 2006). This property also precludes degradation of the misfolded conformer by the proteasome and could explain its preferential accumulation in models of lysosomal dysfunction. However, further studies will be required to elucidate the precise relationship between various types of lysosomal dysfunction and $\alpha$-syn accumulation.

Together with the previous data demonstrating that overexpression of ATP13A2 rescued $\alpha$-syn-mediated toxicity (Gitler et al., 2009), our results suggest that restoration of ATP13A2 function may lead to improved lysosomal function and decreased accumulation of $\alpha$-syn. In broader terms, this work further highlights the importance of lysosomal pathways in the pathogenesis of synucleinopathies and suggests specific mechanistic targets for future development of neuroprotective therapies.

\section{References}

Auluck PK, Chan HY, Trojanowski JQ, Lee VM, Bonini NM (2002) Chaperone suppression of alpha-synuclein toxicity in a Drosophila model for Parkinson's disease. Science 295:865-868.

Cao S, Gelwix CC, Caldwell KA, Caldwell GA (2005) Torsin-mediated protection from cellular stress in the dopaminergic neurons of Caenorhabditis elegans. J Neurosci 25:3801-3812.

Chiti F, Dobson CM (2006) Protein misfolding, functional amyloid, and human disease. Annu Rev Biochem 75:333-366.

Conway KA, Lee SJ, Rochet JC, Ding TT, Williamson RE, Lansbury PT Jr (2000) Acceleration of oligomerization, not fibrillization, is a shared property of both alpha-synuclein mutations linked to early-onset Parkinson's disease: implications for pathogenesis and therapy. Proc Natl Acad Sci U S A 97:571-576.

Cuervo AM, Stefanis L, Fredenburg R, Lansbury PT, Sulzer D (2004) Impaired degradation of mutant alpha-synuclein by chaperone-mediated autophagy. Science 305:1292-1295.

Devine MJ, Gwinn K, Singleton A, Hardy J (2011) Parkinson's disease and $\alpha$-synuclein expression. Mov Disord 26:2160-2168.

Gitler AD, Chesi A, Geddie ML, Strathearn KE, Hamamichi S, Hill KJ, Caldwell KA, Caldwell GA, Cooper AA, Rochet JC, Lindquist S (2009) Alpha-synuclein is part of a diverse and highly conserved interaction network that includes PARK9 and manganese toxicity. Nat Genet 41:308-315.

Hamamichi S, Rivas RN, Knight AL, Cao S, Caldwell KA, Caldwell GA
(2008) Hypothesis-based RNAi screening identifies neuroprotective genes in a Parkinson's disease model. Proc Natl Acad Sci U S A 105:728-733.

Jeong H, Then F, Melia TJ Jr, Mazzulli JR, Cui L, Savas JN, Voisine C, Paganetti P, Tanese N, Hart AC, Yamamoto A, Krainc D (2009) Acetylation targets mutant huntingtin to autophagosomes for degradation. Cell 137:60-72.

Kabeya Y, Mizushima N, Ueno T, Yamamoto A, Kirisako T, Noda T, Kominami E, Ohsumi Y, Yoshimori T (2000) LC3, a mammalian homologue of yeast Apg8p, is localized in autophagosome membranes after processing. EMBO J 19:5720-5728.

Kaushik S, Cuervo AM (2009) Methods to monitor chaperone-mediated autophagy. Meth Enzymol 452:297-324.

Liang C, Lee JS, Inn KS, Gack MU, Li Q, Roberts EA, Vergne I, Deretic V, Feng P, Akazawa C, Jung JU (2008) Beclin1-binding UVRAG targets the class C Vps complex to coordinate autophagosome maturation and endocytic trafficking. Nat Cell Biol 10:776-787.

Martinez-Vicente M, Cuervo AM (2007) Autophagy and neurodegeneration: when the cleaning crew goes on strike. Lancet Neurol 6:352-361.

Masliah E, Rockenstein E, Veinbergs I, Mallory M, Hashimoto M, Takeda A, Sagara Y, Sisk A, Mucke L (2000) Dopaminergic loss and inclusion body formation in alpha-synuclein mice: implications for neurodegenerative disorders. Science 287:1265-1269.

Mazzulli JR, Xu YH, Sun Y, Knight AL, McLean PJ, Caldwell GA, Sidransky E, Grabowski GA, Krainc D (2011) Gaucher disease glucocerebrosidase and $\alpha$-synuclein form a bidirectional pathogenic loop in synucleinopathies. Cell 146:37-52.

Nedelsky NB, Todd PK, Taylor JP (2008) Autophagy and the ubiquitinproteasome system: collaborators in neuroprotection. Biochim Biophys Acta 1782:691-699.

Nixon RA, Yang DS, Lee JH (2008) Neurodegenerative lysosomal disorders: a continuum from development to late age. Autophagy 4:590-599.

Park JS, Mehta P, Cooper AA, Veivers D, Heimbach A, Stiller B, Kubisch C, Fung VS, Krainc D, Mackay-Sim A, Sue CM (2011) Pathogenic effects of novel mutations in the P-type ATPase ATP13A2 (PARK9) causing Kufor-Rakeb syndrome, a form of early-onset parkinsonism. Hum Mutat 32:956-964.

Ramirez A, Heimbach A, Gründemann J, Stiller B, Hampshire D, Cid LP, Goebel I, Mubaidin AF, Wriekat AL, Roeper J, Al-Din A, Hillmer AM, Karsak M, Liss B, Woods CG, Behrens MI, Kubisch C (2006) Hereditary parkinsonism with dementia is caused by mutations in ATP13A2, encoding a lysosomal type 5 P-type ATPase. Nat Genet 38:1184-1191.

Schultz ML, Tecedor L, Chang M, Davidson BL (2011) Clarifying lysosomal storage diseases. Trends Neurosci 34:401-410.

Singleton AB, Farrer M, Johnson J, Singleton A, Hague S, Kachergus J, Hulihan M, Peuralinna T, Dutra A, Nussbaum R, Lincoln S, Crawley A, Hanson M, Maraganore D, Adler C, Cookson MR, Muenter M, Baptista M, Miller D, Blancato J, et al. (2003) alpha-Synuclein locus triplication causes Parkinson's disease. Science 302:841.

Spillantini MG, Schmidt ML, Lee VM, Trojanowski JQ, Jakes R, Goedert M (1997) Alpha-synuclein in Lewy bodies. Nature 388:839-840.

Tan J, Zhang T, Jiang L, Chi J, Hu D, Pan Q, Wang D, Zhang Z (2011) Regulation of intracellular manganese homeostasis by Kufor-Rakeb syndrome-associated ATP13A2 protein. J Biol Chem 286:29654-29662.

Tiscornia G, Singer O, Verma IM (2006) Production and purification of lentiviral vectors. Nat Protoc 1:241-245.

Tresse E, Salomons FA, Vesa J, Bott LC, Kimonis V, Yao TP, Dantuma NP, Taylor JP (2010) VCP/p97 is essential for maturation of ubiquitincontaining autophagosomes and this function is impaired by mutations that cause IBMPFD. Autophagy 6:217-227.

Webb JL, Ravikumar B, Atkins J, Skepper JN, Rubinsztein DC (2003) Alpha-Synuclein is degraded by both autophagy and the proteasome. J Biol Chem 278:25009-25013.

Williams DR, Hadeed A, al-Din AS, Wreikat AL, Lees AJ (2005) Kufor Rakeb disease: autosomal recessive, levodopa-responsive parkinsonism with pyramidal degeneration, supranuclear gaze palsy, and dementia. Mov Disord 20:1264-1271.

Yeger-Lotem E, Riva L, Su LJ, Gitler AD, Cashikar AG, King OD, Auluck PK, Geddie ML, Valastyan JS, Karger DR, Lindquist S, Fraenkel E (2009) Bridging high-throughput genetic and transcriptional data reveals cellular responses to alpha-synuclein toxicity. Nat Genet 41:316-323. 